\title{
Immunisation state of young children admitted to hospital and effectiveness of a ward based opportunistic immunisation policy
}

\author{
D J Riley, M Z Mughal, J Roland
}

\begin{abstract}
Objective-To study the need for and effectiveness of a ward based opportunistic immunisation policy.

Design-A six month prospective study.

Setting-An acute medical paediatric ward of an inner city teaching hospital.

Subjects - 296 children admitted to the ward who lived within Central Manchester Health Authority boundaries and were aged from 5 months to 6 years.

Main outcome measures - Completion of immunisation schedule appropriate for age.

Results -56 children were three or more months behind with immunisations. The parent's history was not reliable for 18 children. Accessing health authority immunisation records was not difficult. The main reasons for falling behind were the mobility of the families ( 15 children), lack of motivation (14), and frequent minor illnesses (9). 40 children were immunised before discharge, but three could not be because of valid contraindications. Of the 16 children requiring more immunisations after discharge, only four obtained them at the correct time and five children not at all.

Conclusion-An opportunistic immunisation policy is an important means of immunising a vulnerable group of children who would often default on routine immunisations, and such policies should operate wherever possible. Our ward based policy can achieve immunisation of three quarters of possible children without change or inconvenience to the daily ward work, but efficacy relies on adequate levels of enthusiastic staff. The system can be improved by having accurate and updated immunisation records available in the hospital, and by encouraging nursing staff to participate.
\end{abstract}

\section{Introduction}

Despite gradual improvement the uptake of Department of Paediatrics, St Mary's Hospital, Manchester M13 0JH

$\mathrm{D} J$ Riley, MB, senior house officer

M Z Mughal, MRCP, consultant

Department of Public Health Medicine, Central Manchester District Health Authority, Manchester J Roland, MFCM, consultant in public health medicine and district immunisation coordinator

Correspondence to: $\mathrm{Dr}$ Mughal.

BMF 1991;302:31-3 central Manchester, fails World Health Organisation of protection of $90 \%$ of the population by the age of 2 years (immunisation statistics supplied by Department of Public Health Medicine, Central Manchester District Health Authority). ${ }^{12}$ Poorly motivated mothers or professionals, or both, confusing or contradictory advice, a highly mobile population, and frequent minor childhood illnesses all contribute to the difficulty of adequately immunising children in these areas. ${ }^{134}$

We believe that such children are not only those less likely to be immunised but also those more likely to be admitted to hospital. A recent survey showed that the immunisation state of children admitted to hospital was not as good as that of those who were attending only outpatient clinics or casualty departments. ${ }^{5}$ The possibility and desirability of identifying and opportunistically immunising such children has been suggested $^{6}$ but considered difficult as it relies on obtaining accurate immunisation records and the parent's history alone is not accurate enough. ${ }^{37}$ The recent Department of Health guidelines on immunisation emphasise that "no opportunity to immunise should be missed."

The aim of our six month study was to determine the following: the immunisation state of young children admitted to the general paediatric ward; the accuracy of the immunisation history given by the parents and its correlation with the data in the immunisation office records; the reasons for the delay if immunisation was overdue; the feasibility of opportunistic immunisation for children who had fallen well behind in their immunisation schedule; whether children immunised in hospital would later complete their course of immunisation at the correct time.

\section{Methods}

STUDY POPULATION

We studied all children who were admitted to the children's medical ward of the hospital from December 1989 to May 1990 inclusive, lived within the boundaries of Central Manchester District Health Authority, were at least three months over the age at which first immunisation was due, and on the ward during working weekday hours. The immunisation schedule used in central Manchester to 30 April 1990 was for the three doses of the primary course of diphtheria, pertussis, tetanus, and polio vaccines to be given at the ages of 3,4 , and 6 months and the measles, mumps, and rubella (MMR) vaccine at 15 months, so all children aged 6 months and over were included in the study. From 1 May 1990 the immunisation schedule was as specified in the Department of Health guidelines $^{8}$ (primary doses at 2, 3, and 4 months), and children aged 5 months and over were then included.

\section{IMMUNISATION RECORD}

The immunisation history was obtained from the

Unless a parent disputed the child health records we assumed these to be correct, and any child identified as being three or more months late in receiving an immunisation was offered the overdue vaccine. The data on the community child health system records were then ascertained by daily direct telephone munication with the Central Manchester Health Authority immunisation office, whose records were Aceekly. The accuracy of personal details (name, date of birth, and address) was also checked The parent's reasons for an immunisation being overdue were obtained by open questioning and the responses grouped accordingly.

\section{IMMUNISATION PROCEDURE}


immunisation was given in accordance with the Department of Health guidelines ${ }^{89}$ when the child was ready for discharge from the ward provided the parents consented and there were no contraindications. It was reported to the health authority as an unscheduled vaccination. Parents were given a written record of the immunisations and told when and where the next one was due. Reference to any immunisation given was included in the discharge letter to the general practitioner. Immunisations were carried out only on working weekdays when both ward clerk and staff at the health authority immunisation records office were available. It took about 10-15 minutes for each to relay the information on the children. Immunisations were carried out by any of the senior house officers on the ward. Each injection, including obtaining consent, counselling, and form filling, took about 15 minutes and there were two or three injections a week.

A minimum of 30 hours without fever after a febrile illness was taken to indicate that immunisation was no longer contraindicated. We did not give the measles, mumps, and rubella vaccine to children who had not received it or had received only the measles vaccine if it could be given at the time of the diphtheria, tetanus, and pertussis booster at school entry. The children who had completed a primary course of immunisation that excluded pertussis for an inappropriate reason were offered advice and immunisation, but their numbers were not recorded in the study. A child who had had one or two doses of diphtheria and tetanus vaccine, however, and then had pertussis antigen included in the opportunistic immunisation was recorded, as were those who had initially completed a course of diphtheria and tetanus immunisation and then started a course of pertussis which they had failed to complete. The children who moved into central Manchester from an authority with a different immunisation schedule were immunised but included in the study only if they were also delayed with their

TABLE I-Reasons given by parents or guardians for delay in their child's immunisation

\begin{tabular}{lc}
\hline Reason & No of children $(\mathrm{n}=56)$ \\
\hline Frequently moving or homeless & 15 \\
Never got round to it & 14 \\
Child always "chesty" & 9 \\
Fear of brain damage & 4 \\
Epilepsy or convulsions in family & 2 \\
Child has history of allergies & 2 \\
Parents refuse immunisations & 2 \\
No specific reason given & 8 \\
\hline
\end{tabular}

^ Resident in local homeless families accommodation.

TABLE II-Numbers of each vaccine that were given, failed to be given, or were contraindicated when children were in hospital

\begin{tabular}{lccc}
\hline Vaccine & No given & $\begin{array}{c}\text { No failed to } \\
\text { be given }\end{array}$ & $\begin{array}{c}\text { No } \\
\text { contraindicated }\end{array}$ \\
\hline Diphtheria/tetanus/pertussis & 21 & 9 & 3 \\
Diphtheria/tetanus & 3 & & 3 \\
Polio & 24 & 9 & \\
Measles/mumps/rubella & 20 & 4 & 6 \\
Pertussis & 8 & 1 & \\
\hline Total & 76 & 23 & \\
\hline
\end{tabular}

TABLE III-Reasons for not immunising children with each of four types of vaccine when they were in hospital

\begin{tabular}{lccccc}
\hline & & \multicolumn{4}{c}{ No of vaccines } \\
\cline { 3 - 6 } Reason & $\begin{array}{c}\text { No of } \\
\text { children }\end{array}$ & $\begin{array}{c}\text { Diphtheria/ } \\
\text { tetanus/pertussis }\end{array}$ & Polio & $\begin{array}{c}\text { Measles/ } \\
\text { mumps/rubella }\end{array}$ & Pertussis \\
\hline Contraindicated & 3 & 3 & 3 & & \\
Parents refused & 2 & 2 & 2 & 1 & \\
Parents refuted health records & 2 & 1 & 1 & 1 & \\
General practitioner wanted to do it & 2 & 2 & 2 & & 1 \\
Transfer to other ward or hospital & 1 & 1 & 1 & 2 & 1 \\
Staff shortage or too busy & 6 & 3 & 3 & 4 & 1 \\
\hline Total & 16 & 12 & 12 & & \\
\hline
\end{tabular}

original schedule. We did not intervene in any cases where the general practitioner had already made arrangements to amend the immunisation deficit.

The study was approved by the Manchester Local Medical Committee.

\section{Results}

In the six months 851 children attended the ward, of whom 296 were eligible for the study. Of these, 56 were at least three months overdue for one or more immunisations. Twelve children needed both diphtheria, tetanus, and pertussis and measles, mumps, and rubella immunisations, the rest requiring only one or other. Eleven had received no immunisations at all, and in all these cases the parents and the immunisation records agreed. In 27 of the remaining 45 cases the parents' history agreed with health authority records; in 11 the parents' history gave more immunisations than were on the records; in three the records showed the child to have had more than the parents knew about; and in four the parents had no idea how many imunisations the child had received. Therefore, the history taken by the doctor at admission was unreliable for 18 children.

The addresses of children on the child health system were incorrect in 35 cases. This did not make tracing the immunisation records difficult because only the child's name and date of birth were needed. In only two cases was the date of birth in the immunisation records incorrect, and in one of these cases the child was completely immunised. The change of surname of some Asian children was not a problem. In only three cases could no trace be found of a child in the records.

Table I shows the reasons that were given by parents for the delay in their child's immunisations in response to direct questioning. The first response given was recorded, but more than one response might apply to one child. Of the 56 children who were delayed in their immunisations when admitted, 40 were immunised before discharge. In three cases there were valid contraindications (fever or diarrhoea), so the immunisation uptake for all possible cases was actually $75 \%$ (40/53). The total number of vaccines given and those missed opportunities is shown in table II. Of a possible 99 non-contraindicated immunisations, 76 were given. Table III shows the reasons for not immunising all possible children.

Of the 40 children immunised, 24 had then completed their necessary immunisations up to date and 16 needed further immunisations at a later date, of which the parents were advised. Of these 16 children, four received their immunisation at the correct time, seven later than they should have, and, at the time of writing (two to six months after they were immunised for the study), five had had no further immunisations. In the first six months of the study we received only one complaint from a general practitioner who thought that his patient should not have been immunised while in hospital.

\section{Discussion}

The size of our study group (296) was almost three times larger than that of a survey in the United States, which also found that a fifth of children admitted to hospital were delayed in their immunisations. ${ }^{10} \mathrm{We}$ chose a delay of three months in immunisation as being long enough to warrant intervention; in fact, possibly for greater benefit an opportunistic policy should operate for any child, however small the delay.

Our findings confirm those of similar studies, which show the unreliability of the parent's history in cases of incomplete immunisation..$^{5}$ Although it has been suggested that parents should carry a medical record 
card for their child, ${ }^{7}$ a recent survey found that such records were available at the presentation of less than half of all admissions. ${ }^{5}$ It may suffice for parents to know only whether or not the immunisation course has been completed if such information is emphasised by the doctor at immunisation. The use of confusing terms such as the three in one injection should be discouraged. We found it more acceptable to both parents and staff if the immunisation history was studied in greater depth once the child's condition had been satisfactorily diagnosed and treated.

When comparing the immunisation history given by parents with that on the child health system we assumed that the system was correct, yet these data too are subject to human error in their recording. Failure to have a child immunised may be the fault of the parent or the health authority. An efficient posted appointments system for routine immunisations requires an accurate and rapidly updated database. In central Manchester there are about 10000 children under 5 years, and about 6000 addresses are changed every year (Department of Public Health, Central Manchester District Health Authority, personal communication). Our results confirm that one of the principal reasons for inadequate immunisation in inner cities is the frequency of moving of some families, who may change address several times in one year. The overall impact of incorrect authority data on immunisation uptake could not be truly assessed because there were cases of completely immunised children whose personal data were incorrect, and there were cases of unimmunised children with their data correctly recorded. Despite deficiencies in the child health system data on addresses it was still possible to access the immunisation details because only name and date of birth are required. Asian children (notably girls) may change their surname but were easily traced when the date of birth was known. ${ }^{11}$ Daily direct telephone communication between the ward clerk and the immunisation records office was not particularly time consuming and was the most inexpensive compromise for accessing information, which has up to now been seen as the main obstacle to opportunistic immunisaction. ${ }^{37}$ Effectively, the child health data were shown to provide sufficiently accurate information for our needs.

Other reasons given for delay were perhaps predictable. Surprisingly often, when directly questioned, a mother would admit to never having got round to taking the child to the clinic because she had other things to do. Many of the children we admitted had (often mild) chest problems and came from home environments that were damp and smokey, and it is not surprising that the mothers tended to consider their child was never well enough to attend for an immunisation. This highlights the need for health professionals to be seen to be keen to immunise children who are suffering minor infections without systemic upset. Hospital doctors, however junior, are readily perceived by parents to be a reliable source of information on the use and safety of immunisations.

At most we immunised three quarters of the possible children despite having a "captive" population. There will always be parents who refuse vaccination altogether, and there were those who maintained, despite our evidence, that their child was satisfactorily immunised. Our study population was a specific subset of the total population of children in the district, a hard core of unimmunised children, and we were not expecting to achieve $100 \%$ uptake. The two children whose general practitioners said that they would bring their immunisations up to date after discharge remain unimmunised.

Under existing conditions we think that we are making an appreciable contribution to the immunisa- tion of a group of vulnerable children who are difficult to reach in the community because of unfavourable social circumstances and whose health may be more at risk. Whereas 240 of these children entered the ward adequately immunised 280 left it immunised. It is sad, therefore, that some families did not take the advice given to them on discharge and failed to obtain further immunisation. Apparently, these children will be immunised only if opportunistic immunisation policies are more widely encouraged in the community and hospitals.

Several recommendations can be made from our findings to improve uptake. Firstly, to overcome the problem of staff shortage we could enlist the help of nursing staff, who, if given the training, could counsel, gain consent, and immunise the child. If we can achieve this we could immunise $87 \%$ of all possible children. Secondly, we could establish liaison with the immunisation record offices of other districts to ascertain the immunisation state of children living outside central Manchester. Thirdly, we could consider children who are seen on the ward but not admitted, and if the ward had a viewer and updated microfiche records as are available in the health authority immunisation office, then we could check the immunisation state of these children and identify many more opportunities for immunisation. Finally, such equipment could also be available in outpatient and casualty departments so that a child's immunisation state could be ascertained as part of the booking in procedure.

We thank our ward clerk, Carol West, and Helen Davies of the immunisation records office. We also thank $\operatorname{Dr} S \mathrm{~W}$ D'Souza, Professor R Boyd, Dr F Bamford, Dr M L Chiswick, and Dr D G Sims for allowing children in their care to participate in the study.

1 Jarman B, Bosanquet N, Rice P, Dollimore N, Leese B. Uptake of immunisation in district health authorities in England. $B M \mathcal{F}$ 1988;296: 1775-8

2 Begg NT, Noah ND. Immunisation targets in Europe and Britain. $B M \mathcal{J}$ 1985;291:1370-1.

3 Nicoll A, Elliman D, Begg N. Immunisation: causes of failure and strategies and tactics for success. BMF 1989;299:808-12.

4 James J, Clark C, Rossdale M. Improving health care delivery in an inner city well baby clinic. Arch Dis Child 1986;61:630.

5 Ferson MJ. Immunisation state and its documentation in hospital patients. Arch Dis Child 1990;65:763-7.

6 Fulginiti VA. Incomplete immunisation, hospitalisation and speciality care. An opportunity to improve the immunisation status of very young children.

Am $\mathcal{F}$ Dis Child 1988;142:704.
7 Hull D. Immunising children in hospital. BMF 1988;297:755.

8 Department of Health and Social Security. Immunisation against infectious diseases. London: HMSO, 1990.

9 Department of Health and Social Security. Immunisation against infectious diseases. London: HMSO, 1988

10 Tifft CJ, Lederman HM. Immunisation status of hospitalised pre-school age children. Am f Dis Child 1988;142:719-20.

11 Black J. The new paediatrics: child health in ethnic minorities. London: British Medical Journal, 1985.

(Accepted 12 November 1990)

\section{ANY QUESTIONS}

Is quinidine more effective than quinine in treating or preventing nocturnal cramps?

I have not been able to find any studies comparing the efficacy of quinine and quinidine in preventing cramp. Quinidine is the dextroisomer of quinine and has the same pharmacological actions on skeletal muscle. Both drugs increase the refractory period of skeletal muscle and have a curare-like effect on the motor end plate. I would expect them to have similar efficacy in preventing cramp. Quinidine has a greater effect on the heart than quinine and, in view of its potential cardiotoxicity, it seems sensible to prefer quinine for the prevention of cramp. - LINDA BEELEY, director, drug and therapeutics unit, Birmingham 